\title{
Mutaciones del gen del receptor 3 del Factor de Crecimiento de Fibroblasto (FG FR3) en pacientes chilenos con talla baja idiopática, hipocondroplasia y acondroplasia
}

\author{
Edna E Mancilla V ${ }^{1}$, Helena Poggi $\mathbf{M}^{3 a}$, \\ Gabriela Repetto $L^{1}$, Cristian García $B^{1,2}$, \\ Arnaldo Foradori $\mathbf{C}^{3}$, Andreina Cattani $0^{1}$. \\ Mutations in the Fibroblast Growth Factor \\ Receptor 3 gene (FG FR3) in Chilean patients \\ with idiopathic short stature, \\ hypochondroplasia and achondroplasia
}

\begin{abstract}
Background: Achondroplasia and hypochondroplasia are skeletal dysplasias of autosomal dominant inheritance that represent different degrees of severity of the same pathological entity. Both dysplasias are caused by mutations in the Fibroblast Growth Factor Receptor 3 (FGFR3) gene. In achondroplasia more than $95 \%$ of the cases studied to date carry the same mutation (G380R). Hypochondroplasia represents a greater clinical and genetic heterogeneity, possibly being confused with "idiopathic short stature". The N540K mutation has been detected in 50$70 \%$ of cases of hypochondroplasia and mutations at the 650 locus in approximately $2.8 \%$. Aim: To assess the frequency of N540K and G380R mutations, and changes at the 650 locus in Chilean patients with idiopathic disproportionate short stature, hypochondroplasia and achondroplasia. Patients and Methods: We studied 21 patients referred for idiopathic short stature, 5 with clinically suspected hypochondroplasia and 4 with achondroplasia. The G1138A, G1138C (G380R), and C1620, C1620A (N540K) mutations and the nucleotide changes at the 650 locus were studied using PCR and restriction analysis of genomic DNA. Results: Three out of five hypochondroplasia patients were heterozygous for the N540K mutation. All of the 4 patients with achondroplasia presented the G1138A mutation. None of these mutations were found in patients with idiopathic short stature. Conclusion: Chilean patients with hypochondroplasia and achondroplasia have the same mutations described in other ethnic groups. The identification of mutations in 3 out of 5 patients with hypochondroplasia shows that this analysis is a useful tool for its diagnostic confirmation. In short stature the molecular study should only be indicated in those cases presenting other clinical and/or radiological features of hypochondroplasia (Rev Méd Chile 2003; 131: 1405-10).
\end{abstract}

(Key Words: Achondroplasia; Genetic, medical; Hypochondroplasia; Receptors, fibroblast growth factor)

Recibido el 10 de abril, 2003. Aceptado en versión corregida el 7 de agosto, 2003.

Departamentos de Pediatría ${ }^{1}$ y Radiología 2 , Servicio de Laboratorios Clínicos 3 , Facultad de Medicina, Pontificia Universidad Católica de Chile.

aioquímica

Correspondencia a: Dra. Edna E Mancilla V. Programa de Fisiología y Biofísica, Facultad de Medicina, ICBM, Universidad de Chile. Independencia 1027, Santiago. Fono: 6786038. Fax: 7776916. E-mail: emancill@med.uchile.cl 
Las as displasias óseas constituyen un problema en el diagnóstico diferencial del niño con talla baja, en especial aquellas formas clínicamente menos evidentes, como la hipocondroplasia. Dentro de las displasias óseas, la más frecuente es la acondroplasia, con una incidencia estimada de 15 a 40 en 100.000 nacimientos. Se cree que la incidencia de la hipocondroplasia se acerca a este número, pero es difícil de establecer dada la dificultad del diagnóstico clínico y radiológico.

La acondroplasia y la hipocondroplasia representan distintos grados de severidad de una misma patología. La primera se caracteriza por un enanismo de predominio rizomélico, talla baja de nacimiento y una serie de dismorfias, entre las cuales destacan: macrocefalia, hipoplasia de la región maxilar, acortamiento de los huesos largos y dedos, y radiológicamente, platispondilia, disminución de la distancia interpedicular de la columna lumbar, deformidad de las regiones metafiso-epifisiarias, huesos ilíacos cuadrados y marcadamente disminuidos de altura y un foramen magnum pequeño, entre otras. El diagnóstico fenotípico es evidente en cualquier etapa de la vida, incluso en ocasiones durante el período prenatal $^{1-5}$.

La hipocondroplasia, en cambio, es una displasia ósea más leve, con talla de nacimiento generalmente dentro del rango normal, acortamiento de extremidades menos marcado, talla final mayor y sin las características faciales de la acondroplasia. La sutileza de las manifestaciones observadas en la hipocondroplasia, junto con una talla final mayor que la encontrada en acondroplasia hacen que ésta pueda confundirse con una talla baja idiopática. Las características radiológicas más frecuentes de esta entidad, descritas por Hall y Spranger en 1979, incluyen falta de progresión 0 disminución en la distancia interpedicular lumbar, acortamiento de los huesos ilíacos, huesos largos cortos y engrosados en grado variable, a veces con irregularidad metafisiaria, acortamiento difuso y simétrico de los huesos tubulares de las manos, elongación relativa del extremo distal de los peronés, acortamiento relativo del extremo distal de los cúbitos ${ }^{3}$. Prinster et al estudiaron las características radiológicas en pacientes con hipocondroplasia, portadores y no portadores de la mutación N540K, concluyendo que las alteracio- nes radiológicas más frecuentes en ambos casos correspondían a aquellas de la columna lumbar y de los peronés 4 .

Ambas condrodisplasias son de herencia autosómica dominante, siendo la mayoría de los casos causados por mutaciones ocurridas de novo. La causa molecular de la acondroplasia fue inicialmente ligada a la región cromosómica $4 p$ y luego identificada como una mutación en el gen del Receptor del Factor de Crecimiento de Fibroblasto 3, FGFR3. En más de 95\% de los casos de acondroplasia estudiados hasta la fecha, se describe la misma mutación activante, una transición de $\mathrm{G}$ a A en el nucleótido 1138 del ADNc, resultando en una sustitución de glicina por arginina en la posición 380 de la proteína en la región transmembrana del receptor ${ }^{6-12}$.

Con el hallazgo de mutaciones en FGFR3 en pacientes con diagnóstico clínico de hipocondroplasia se confirmó que ambas displasias compartían la etiología genética. En $50-70 \%$ de los casos de hipocondroplasia estudiados hasta la fecha se han encontrado las mutaciones C1620A y C1620G, ambas produciendo la sustitución N540K, ubicada en el dominio tirosina-quinasa 1 de la proteína ${ }^{13,14}$. En un menor número de casos $( \pm 2,8 \%)$ con fenotipos más sutiles, se han encontrado mutaciones en el codón 650 del exón 15 de FGFR3, y en familias aisladas se han descrito las mutaciones I538V, N540T, N328I y N540S. Se cree que en aproximadamente $27 \%$ de los pacientes existe heterogeneidad de locus, con exclusión de ligamiento a $4 \mathrm{p} 16.3^{15-20}$.

Nuestros objetivos fueron estudiar la presencia de las mutaciones N540K, N650 y G380R en pacientes chilenos que cumplían con los criterios clínicos y radiológicos de acondroplasia e hipocondroplasia, y evaluar su utilidad en el diagnóstico diferencial de pacientes con talla baja aparentemente idiopática.

\section{PACIENTES y MÉTOdOS}

Se incluyeron en el estudio todos los pacientes con talla baja idiopática con algún grado de desproporción de segmentos corporales y aquellos clasificados con el diagnóstico clínico de acondroplasia o hipocondroplasia que fueron 
referidos a nuestro hospital entre noviembre de 1999 y noviembre de 2000. El protocolo se realizó bajo las normas éticas de Helsinki y no tuvo objeciones por parte del Comité de Ética de la Facultad de Medicina de la Pontificia Universidad Católica de Chile.

Los criterios de inclusión para los pacientes con talla baja idiopática fueron: puntaje $\mathrm{Z}$ de talla menor de -2 y relación de segmentos corporales (superior vs inferior) entre +1 y $+2 \mathrm{DE}$, utilizando gráficos de McKusick et $\mathrm{al}^{21}$. En todos ellos se había descartado previamente patologías endocrinas o metabólicas.

Los pacientes referidos con diagnóstico clínico de hipocondroplasia tenían puntaje $\mathrm{Z}$ de talla menor a -2 , relación de segmentos corporales mayor a +2 DE y presentaban a lo menos dos características fenotípicas tales como: macrocefalia relativa, braquidactilia y rizomelia/mesomelia 0 alteraciones radiológicas, siendo lo más frecuente, una falta de progresión o estrechamiento en la distancia interpedicular lumbar. Los pacientes con acondroplasia tenían un diagnóstico evidente por su fenotipo.

Se obtuvo una muestra de sangre periférica de cada paciente, de la cual se extrajo ADN genómico por el método de Lahiri. En todos los pacientes se estudiaron las mutaciones G1138A y G1138C, C1620A y C1620G, así como los cambios en el codón 650.

Amplificación: Para el estudio de acondroplasia se amplificó por PCR el exón 10 y para hipocondroplasia los exones 13 y 15 con partidores ya descritos $^{8,13,15}$. El PCR se llevó a cabo en un volumen final de $50 \mu \mathrm{L}$ con una mezcla de reacción que contenía: partidores $0,5 \mu \mathrm{M}$, buffer de reacción (10 mmol Tris- $\mathrm{HCl}, 50 \mathrm{mmol} \mathrm{KCl}), 1,5$ mmol $\mathrm{MgCl}_{2}, 0,2 \mathrm{mmol}$ dNTPs, $2 \mathrm{U}$ de Taq polimerasa, $10 \%$ DMSO y $5 \mu \mathrm{L}$ de ADN genómico (50-150 ng/ $\mu \mathrm{l})$. El programa de amplificación para los 3 PCR consistió en: una denaturación inicial de $94^{\circ} \mathrm{C}$ por $5 \mathrm{~min}, 35$ ciclos de $95^{\circ} \mathrm{C}$ por $30 \mathrm{~s}, 60^{\circ} \mathrm{C}$ por $30 \mathrm{~s}, 72^{\circ} \mathrm{C}$ por $30 \mathrm{~s}$ y una extensión final a $72^{\circ} \mathrm{C}$ por 5 min. El producto de PCR se analizó en geles de agarosa al 1\%, comprobándose la presencia de productos de 164, 578 y 335 bp correspondientes a los exones 10, 13 y 15, respectivamente.

Digestión: Todas las digestiones se realizaron con $10 \mathrm{U}$ de enzima (excepto $20 \mathrm{U}$ para AluI) a $37^{\circ} \mathrm{C}$ durante alrededor de 18 h, analizándose los productos de digestión en geles de agarosa NuSieve al 3\%. Las mutaciones G1138A y G1138C presentes en acondroplasia fueron estudiadas con las enzimas ScfI y MspI que crean sitios de restricción. En un sujeto heterocigoto para la mutación G1138A, al digerir con ScfI, se observan fragmentos de 109 y 55 bp, más allá del producto no digerido de $164 \mathrm{bp}$, mientras que en heterocigotos G1138C, al digerir con MspI, se observan fragmentos de 107 y 57 bp (más 164 bp). Para el estudio de hipocondroplasia se digirió el exón 13 con BspMI, por lo que en un sujeto heterocigotoADN para la mutación C1620A o C1620G se pierde un sitio de restricción y se encuentra un fragmento de $514 \mathrm{bp}$ (además de los de 367, 147 y $64 \mathrm{bp}$ ). Al digerir este exón con AluI se gana un sitio de restricción cuando está presente el cambio C1620G y se observan fragmentos de 339 y 239 bp (además del de 578 bpy 64 bp). Alteraciones en el codón 650 del exón 15 generan la pérdida del sitio de restricción de la enzima BbsI, por lo que en un sujeto heterocigoto se encuentran un fragmento de $335 \mathrm{bp}$, además de los de 230 y 130 bp. En este codón es posible encontrar cambios en cualquiera de sus tres bases, generándose distintos fenotipos por lo que al perderse el sitio de restricción se debe secuenciar el producto de PCR para identificar qué mutación está presente ${ }^{15}$.

\section{RESULTADOS}

Durante el período de estudio evaluamos 21 pacientes con diagnóstico de talla baja idiopática definida según los criterios ya detallados. De ellos, 12 eran varones y 9 mujeres con edades que fluctuaban entre 2 y 14 años. Además evaluamos a 5 pacientes en quienes se sospechó clínicamente una hipocondroplasia con edades de 2 a 38 años, e incluimos en el estudio a 4 pacientes con diagnóstico clínico de acondroplasia.

En los pacientes con talla baja idiopática no se detectó ninguna de las mutaciones estudiadas, aun en aquellos que además de la talla baja, tenían un criterio diagnóstico adicional de hipocondroplasia.

En los pacientes con hipocondroplasia se detectaron mutaciones en 3 de 5 pacientes, dos fueron heterocigotos para la mutación C1620G y 
Tabla 1. Características clínicas y mutaciones en pacientes con hipocondroplasia

\begin{tabular}{|lcccccc|}
\hline Edad & Sexo & $\begin{array}{c}\text { Puntaje } \\
\text { Z talla }\end{array}$ & SS/SI* & Fenotipo & RX & Mutación \\
\hline 4a 6m & M & $-3,3$ & $>+2$ DE & MC, B & No tiene & C1620G/wt \\
19a 8m & M & $-5,5$ & $>+2$ DE & MC, B, MM & AE** & C1620G/wt \\
2a 10m & F & $-3,0$ & $>+2$ DE & MC, RM, B & FPL, AE & C1620A/wt \\
38a & F & $-3,5$ & $>+2$ DE & MC, B, MM & Normal & $\mathrm{wt} / \mathrm{wt}$ \\
8a 7m & F & $-5,1$ & $>+2$ DE & MC, B, RM & FPL, AE & $\mathrm{wt} / \mathrm{wt}$ \\
\hline
\end{tabular}

MC: Macrocefalia, B: Braquidactilia, RM: Rizomelia, M: Mesomelia, FPL: Falta de progresión lumbar, AE: Acortamiento y engrosamiento huesos tubulares.

*Handbook of Normal Physical Measurements, p 220-287, Ed Oxford Univ. Press **RX columna no disponible. wt wildtype, alelo no afectado por las mutaciones.

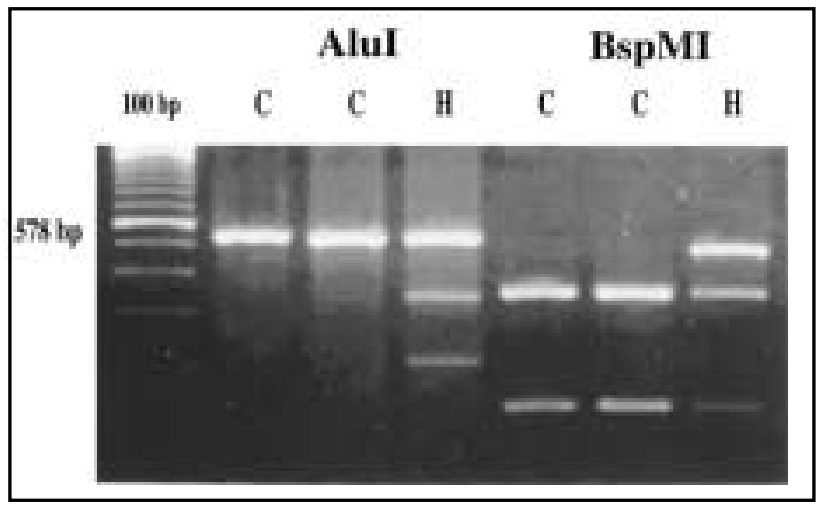

FiguRA 1. Gel representativo del análisis de la mutación N540K en hipocondroplasia. Columna 1: Estándar de peso molecular. Columnas 2, 3: Digestión con AluI en controles normales sólo se observa producto de 578 bp. Columna 4: Digestión con AluI en paciente con la mutación C1620G, se observa producto de 578 bp y fragmentos de 339 y 239 bp. Columnas 5 y 6 : Digestión con enzima BspMI en controles normales fragmentos de 367 y $147 \mathrm{bp}$. Columna 7: Digestión con enzima BspMI en paciente con la mutación C1620A, se observan fragmentos de 514 bp, 367 bp y 147 bp.

uno para la mutación C1620A (Tabla 1). La Figura 1 muestra un gel representativo con los productos de digestión. El primer caso con la mutación C1620G detectado por nosotros fue confirmado por secuenciación automática. La mutación C1620A había sido previamente diagnosticada en el laboratorio de diagnóstico molecular de University of Colorado Health Sciences Center. En nuestra casuística no se detectaron cambios en el codón 650. En 4 de 5 pacientes se pudieron obtener las radiografías diagnósticas y en 3 de ellos pudimos evaluar radiografía de columna lumbar. No se observaron diferencias fenotípicas entre los pacientes con hipocondroplasia portadores de la mutación y los que no la presentaron (Tabla 1).

Todos los pacientes con acondroplasia fueron heterocigotos para la mutación G1138A. En la Figura 2 se muestra un gel representativo mostrando la digestión con Scfl en 2 casos con la mutación G1138A.

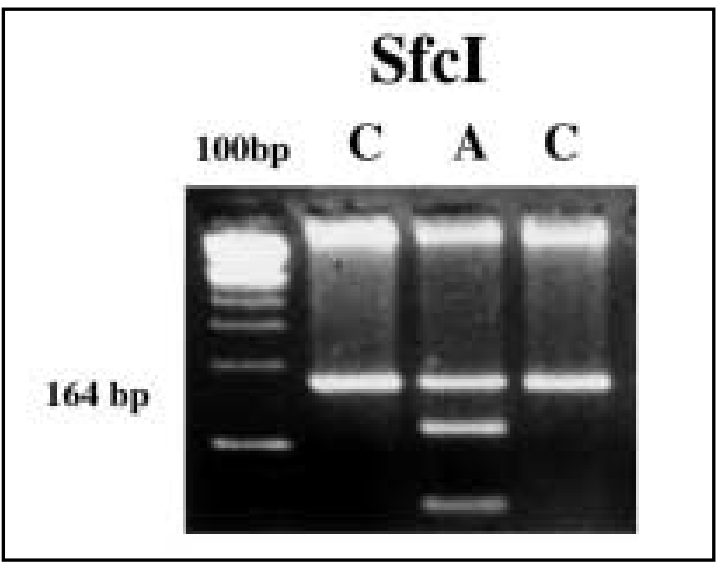

FIgURA 2. Gel representativo de la digestión con SfCI para el estudio de la mutación G1138A en acondroplasia. Columna 1: Estándar de peso molecular. Columna 2 y 4: Controles normales con sólo producto de $164 \mathrm{bp}$. Columna 3: Paciente con acondroplasia con fragmentos de 164, 109 y $55 \mathrm{bp}$. 


\section{DiSCUSIÓN}

Este estudio representa el primer análisis genéticomolecular en pacientes con acondroplasia e hipocondroplasia en población chilena, confirmándose que en $95-100 \%$ de los pacientes con acondroplasia se encuentra la mutación G380R y que la mutación predominante en hipocondroplasia es N540K.

Nuestro estudio confirmó que la etiología molecular de estas displasias óseas en población chilena es semejante a la de otras etnias. En los casos de acondroplasia, siendo el diagnóstico fenotípicamente evidente, no es necesario confirmar el diagnóstico con análisis molecular. Sin embargo, siendo la hipocondroplasia una displasia esquelética leve, con una talla final entre $127 \mathrm{y}$ $152 \mathrm{~cm}$ y características clínicas y radiológicas sutiles, especialmente en los primeros años de vida, el estudio genético-molecular es un elemento de gran utilidad en la confirmación diagnóstica. Dado que la hipocondroplasia es de herencia autosómica dominante, la confirmación molecular de esta enfermedad es también de utilidad para el consejo genético de los pacientes.

\section{REFERENCIAS}

1. Paul RM. Osteochondrodysplasias with Mild Clinical Manifestations: A Guide for Endocrinologists and Others. Growth 1995; 11: 1-5.

2. Vajo S, Francomano CA, Wilkin DJ. The Molecular and Genetic Basis of Fibroblast Growth Factor Receptor 3 Disorders: The Achondroplasia Family of Skeletal Dysplasias, Muenke Craniosynostosis, and Crouzon Syndrome with Achantosis Nigricans. Endocrine Reviews 2000; 21: 23-39.

3. Hall BD, Spranger J. Hypochondroplasia: Clinical and Radiological Aspects in 39 Cases. Radiology 1979; 133: 95-100.

4. Prinster C, Del Maschio M, Beluffi G, Maghnie M, Weber G, Del Maschio A et al. Diagnosis of Hypochondroplasia: the Role of Radiological Interpretation. Pediatr Radiol 2001; 31: 203-8.

5. Wynne-Davies R, Walsh WK, Gormey J. Achondroplasia and Hypochondroplasia. J Bone Joint Surg 1981; 63B: 508-15.
Es importante destacar que dado el porcentaje de detección de mutaciones que se alcanza en hipocondroplasia (50-70\%), un estudio negativo no la descarta. Diversos trabajos con análisis de un mayor número de pacientes de diferentes etnias, han mostrado que en aproximadamente un tercio de los pacientes que cumplen criterios clínicos estrictos de hipocondroplasia, no se ha encontrado la base molecular,22-25, así como tampoco han podido establecer una relación genotipo/fenotipo concluyente.

Con el fin de pesquisar formas más leves de hipocondroplasia, nosotros utilizamos criterios amplios en la selección de pacientes con talla baja idiopática y no encontramos mutaciones en ellos. Sin embargo, por nuestro reducido número de casos no podemos emitir conclusiones con respecto a su utilidad en tallas bajas idiopáticas con dismorfias sutiles. Por lo anterior, nosotros sugerimos indicar el estudio de estas mutaciones sólo en casos de talla baja con puntaje Z menor a -2, desproporción de segmentos mayor a $+2 \mathrm{DE}$ y que presenten otras características fenotípicas o radiológicas de hipocondroplasia.

6. Francomano CA, Ortiz de Luna RI, Hefrerson tW, Belus GA, Turner CE, Taylor E et al. Localization of the acondroplasia gene to the distal $2.5 \mathrm{Mb}$ of human chromosome 4p. Hum Mol Genet 1994; 3: 787-92.

7. Le Merrer M, Rousseau F, Legeai-Malet L, Landais JC, Pelet A, Bonaventure J et al. A gene for achondroplasia-hypochondroplasia maps to chromosome 4p. Nat Genet 1994; 6: 314-7.

8. Shiang R, Thompson LM, Zhu YZ, Church DM, FIELDER TJ, Bocian M ET aL. Mutations in the Transmembrane Domain of FGFR3 Cause the Most Common Genetic Form of Dwarfism, Achondroplasia. Cell 1994; 78: 335-42.

9. Stoilov I, Kilpatrick MW, Tsipouras P. A Common FGFR3 Gene Mutation is Present in Achondroplasia but not in Hypochondroplasia. Am J Med Genet 1995; 55: 127-33.

10. Wang TR, Wang WP, Hwu WL, Lee ML. Fibroblast Growth Factor 3 (FGFR3) Gene G1138A Mutation in Chinese Patients with Achondroplasia. Hum Mut 1996; 8: 178-9. 
11. Bonaventure J, Rousseau F, Legeai-Malet L, Le Merrer M, Munnich A, Maroteaux P. Common mutations in the Fibroblast Growth Factor Receptor 3 (FGFR3) Gene Account for Achondroplasia, Hypochondroplasia and Thanatophoric Dysplasia. Am J Med Genet 1996; 63: 148-54.

12. Ezquieta Zubicaray B, Iguacel AO, Vareia Junquera JM, Jariego Fente CM, GonZalez-Gancedo P, GraciaBOUTHELER R. Gly380Ang and Asn540Lys mutations of fibroblast growth factor receptor 3 in achondroplasia and hypochondroplasia in the Spanish population. Med Clin (Barc) 1999; 112: 290-3.

13. Beluus GA, Mcintosh I, Smith EA, Aylsworth AS, Kaitila I, Horton WA et AL. A Recurrent Mutation in the Tyrosine Kinase Domain of Fibroblast Growth Factor Receptor 3 Causes Hypochondroplasia. Nature Genetics 1995; 10: 357-9.

14. Prinos P, Costa T, Sommer A, Kilpatrick MW, Tsipouras P. A Common FGFR3 Gene Mutation in Hypochondroplasia. Hum Mol Genet 1995; 4: 2097-101.

15. Beluus GA, Spector EB, Speiser PW, Weaver CA, Garber AT, Bryke CR et AL. Distinct Missense Mutations of the FGFR3 Lys650 Codon Modulate Receptor Kinase Activation and the Severity of the Skeletal Dysplasia Phenotype. Am J Hum Genet 2000; 67: 1411-21.

16. Grigelioniene G, Hagenas L, Eklof O, Neumeyer L, Haereid PE, Anvret M. A Novel Missense Mutation Ile538Val in the Fibroblast Growth Factor Receptor 3 in Hypochondroplasia. Hum Mutat 1998; 11: 333.

17. Deutz-Terlouw PP, Losekoot M, Aalfs CM, HenNEKAM RC, BAKKER E. Asn540Thr Substitution in the Fibroblast Growth Factor Receptor 3 Tyrosine Kinase Domain Causing Hypochondrplasia. Hum Mutat 1998; Suppl 1 S62-S65.
18. Mortier G, Nuytinck L, Craen M, Renard JP, Leroy JG, Depaepe A. Clinical and Radiological Features of a Family with Hypochondroplasia Owing to a Novel Asn540Ser Mutation in the Fibroblast Growth Factor Receptor 3 Gene. J Med Genet 2000; 37: 220-4.

19. Winterpatch A, Hilbert K, Stelzer C, Schweikardt T, Decker $H$, Segerer et al. A novel Mutation in FGFR-3 Disrupts a Putative Glycosylation Site and Results in Hypochondroplasia. Physiol Genomics 2000; 2: 9-12.

20. Stoilov I, Kilpatrick MW, Tsipouras P, Costa T. Possible Genetic Heterogeneity in Hypochondroplasia. J Med Genet 1995; 32: 492-3.

21. McKusick VA. Heritable Disorders of Connective Tissue, CV Mosby, 1972.

22. Prinster C, Carrera P, Del Maschiom M, Weber G, Maghnie M, Vigone MC ET AL. Comparison of Clinical-Radiological and Molecular Findings in Hypochondroplasia. Am J Med Genet 1998; 75 : 109-12.

23. Rousseau F, Bonaventure J, Legeai-Malet L, Schmidt $\mathrm{H}$, Weissenbach J, Maroteaux P et al. Clinical and genetic heterogeneity of Hypochondroplasia. J Med Genet 1996; 33: 749-52.

24. Ramaswami U, Rumsby G, Hindmarsh PC, Brook CGD. Genotype and Phenotype in Hypochondroplasia. J Pediatr 1998; 133: 99-102.

25. Matsui $\mathrm{Y}$, Yasui $\mathrm{N}$, Kimura $\mathrm{T}$, Tsumaki N, Kawabata $\mathrm{H}, \mathrm{OcHI}$ T. Genotype phenotype correlation in acondroplasia and hypochondroplasia. J Bone Joint Surg 1998; 80-B: 1052-6.

Agradecimientos

Se agradece a los doctores Marta Arriaza, Hernán García y Verónica Mericq, por derivar pacientes para el estudio. 